\title{
Application of Gel Electrophoresis Techniques to the Study of Wine Yeast and to Improve Winemaking
}

\author{
María Esther Rodríguez, Laureana Rebordinos, Eugenia Muñoz-Bernal, \\ Francisco Javier Fernández-Acero and Jesús Manuel Cantoral \\ Microbiology Laboratory, Faculty of Marine and Environmental Sciences \\ University of Cadiz, Puerto Real \\ Spain
}

\section{Introduction}

Yeasts are unicellular fungi that are frequently used as a model and tools in basic science studies. This is the case of the laboratory yeasts Saccharomyces cerevisiae, which were introduced in the laboratory for genetics and molecular studies in about 1935. There is, however, a second type of yeast comprising those used in industrial processes, for example, in brewing, baking and winemaking. Wine yeast and its properties have been known to humans for as long as civilizations have existed, and the earliest evidence of this yeast has been dated to Neolithic times (Mortimer, 2000).

Most wine yeast strains are diploid and have a low frequency of sporulation. Another important characteristic of wine yeasts, and those used in other industries, is their highly polymorphic chromosomes: their genetic constitution is affected by the frequent and extensive mutation they undergo. These effects include (i) aneuploidy, (ii) polyploidy, (iii) amplification and deletion of chromosomal region or single gene, and (iv) the presence of hybrid chromosomes. The chromosomal polymorphism obtained by applying the technique known as pulsed field gel electrophoresis (PFGE) has been used to characterize and to classify strains that belong to the same species.

In the wine industry, knowledge of the yeast species responsible for the alcoholic fermentation is important because these yeasts with their metabolism contribute significantly to the organoleptic characteristics of the finished wine (Fleet, 2008). The diverse range of yeasts associated with the vinification process can be classified in two groups. The first group is formed principally by the genera Hanseniaspora, Torulaspora, Metschnikowia, Candida, Zygosaccharomyces, etc. These yeasts initiate spontaneous alcoholic fermentation of the must, but they are soon replaced by the second group, formed by Saccharomyces yeasts, which are present during the subsequent phases of the fermentation until it is completed. Within the genus Saccharomyces the species most relevant for the fermentation process are $S$. cerevisiae and S. bayanus var. uvarum; this is because they have become of interest for their biotechnological properties. However, there is currently increasing interest in the nonSaccharomyces yeasts for the development of innovative new styles of wine (Viana et al., 
2009). In the industry, knowledge of specific strains of these microorganism species is important for (i) their selection; (ii) their use as starter cultures; and (iii) improving the fermentation process.

During the 1990's the development of molecular techniques has enabled the identification and characterization of different strains belonging to the same species of yeast, and it has been possible to establish the ecology of spontaneous fermentations in many of the world's winemaking regions (Fleet, 2008). These techniques also constitute a powerful tool not only for the selection of the most suitable yeast, since they tell us which yeasts are the most representative in the fermentation process, but also for obtaining information on the addition to the must of particular strains of yeast in the case of inoculated fermentations (Rodríguez et al., 2010).

Two of the approaches most often used for the molecular characterization of industrial yeast are analysis of the electrophoretic karyotypes by pulsed-field gel electrophoresis (PFGE) and analysis of the restriction fragment length polymorphism of the mitochondrial DNA (mtDNA-RFLP). We have used PFGE in winemaking to analyse the diversity of wild yeasts in spontaneous fermentation of a white wine produced in a winery in SW Spain with the object of selecting the most suitable autochthonous starter yeast; and from the results of the inoculation, we were able to make decisions for improving the efficiency of the process and to establish procedures for the proper performance of the inoculation (Rodríguez et al., 2010). We have also applied the analysis of the karyotypes to characterize natural yeasts in biodynamic red wines in another region of Spain. In this chapter we also evaluate the use of the mtDNA-RFLP technique for quick monitoring of the dominance of inoculated strains in industrial fermentation, without any need for the prior isolation of yeast colonies (Rodríguez et al., 2011).

Another electrophoretic technique has been used to show substantial changes in protein levels in selected wine yeasts under specific growth conditions. It has recently been stated that the proteome is "the relevant level of analysis to understand the adaptations of wine yeasts for fermentation" (Rossignol et al., 2009). Following this, in-depth studies are now being made of the proteome of wine yeast strains and the relationship between the proteome and wine quality and winery processes. We are now exploring more generally the relevance of proteomics to wine improvement. In this chapter, we will summarize the efforts being made by the proteomics research community to obtain the knowledge needed on proteins in the post-genomics era

\section{Pulsed-field gel electrophoresis (PFGE) for the study of yeast population}

PFGE as a system encompasses a series of techniques in which the intact chromosomes of microorganisms like yeasts and filamentous fungi are submitted to the action of a pulsing electric field in two orientations that is changing direction, in a matrix of agarose. The bestknown PFGE modality is the CHEF system (Contour-clamped Homogeneous Electric Fields); this consists of a hexagon of 24 electrodes surrounding the gel that produce a homogeneous electric field alternating between two directions orientated at $120^{\circ}$ with respect to each other. Using this system, Chu et al. (1986) resolved the electrophoretic karyotype of Saccharomyces cerevisiae in 15 bands in a size range of 200-2200 kb. Before performing the electrophoresis the yeast cells must be suitably treated, avoiding the direct 
manipulation of the genetic material to prevent possible rupture of the chromosomes. The cells are then embedded in blocks of agarose which are subsequently treated with a reducing agent and $\mathrm{K}$ proteinase to destabilize the wall and cytoplasmatic membranes, respectively (Figure 1), thus facilitating the release of the DNA when submitted to the action of an electric field.

This methodology for correctly obtaining the karyotype of S. cerevisiae is based on the procedure described by Carle \& Olson (1985) and optimized by Rodríguez et al. (2010). It also depends on the concentration of the agarose gel $(1 \%)$, buffer $(0.5 \times \mathrm{TBE})$, initial and final switch (60-120 seconds respectively), run time (24 hours), voltage $(6 \mathrm{~V} / \mathrm{cm})$ and buffer temperature $\left(14^{\circ} \mathrm{C}\right)$.

The analytical results given by this technique are the number and size of the yeast chromosomes, and it allows specific strains of Saccharomyces to be differentiated because their karyotypes show distinct bands running below the 500-kb marker. It also allows the differentiation between $S$. cerevisiae and Saccharomyces bayanus var. uvarum (S. uvarum) species (Naumov et al., 2000, 2002).

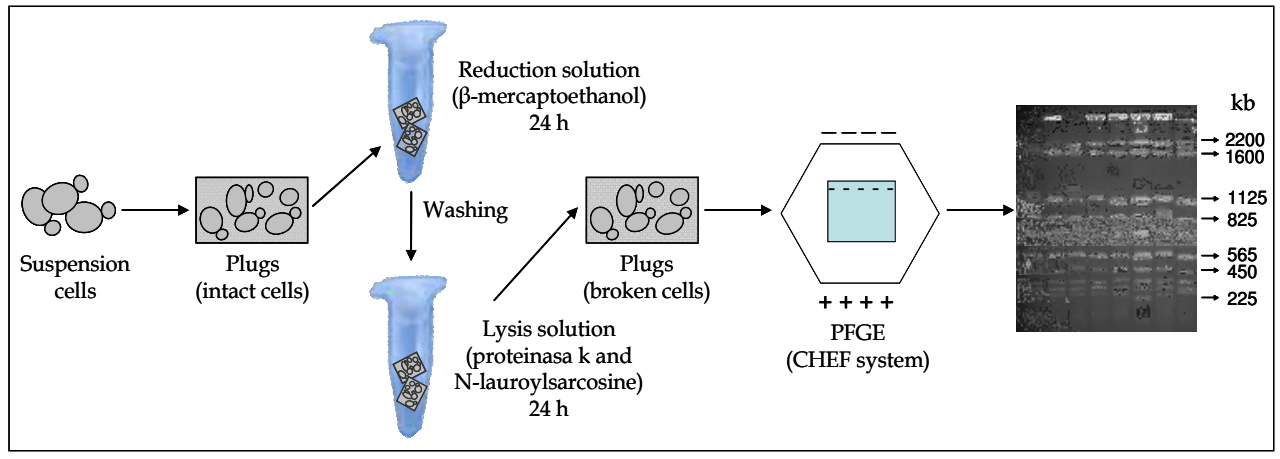

Fig. 1. Methodology for characterizing yeast strains, using pulsed field gel electrophoresis to obtain the karyotype.

In previous research the PFGE technique has been used to analyse the dynamics of the yeast population during the spontaneous fermentations of wine (Demuyter et al., 2004; Martínez et al., 2004; Naumov et al., 2002; Raspor et al., 2002; Rodríguez et al., 2010), and it has also been used to characterize other industrial yeasts including baker's and brewer's yeast (Codón et al., 1998). Another relevant application of PFGE has been to characterize the yeast population which is present in the flor velum that grows on the surface of fino-type sherry wines in the barrel, during their biological ageing process (Mesa et al., 1999, 2000). The results revealed an interesting correlation between the yeast genotypes and the different blending stages.

One disadvantage of the technique is that it is laborious, expensive and requires specialized personnel; increasingly, therefore, analysts are resorting to other simpler and faster techniques to discriminate between yeast clones, like, for example, interdelta analysis of sequences or microsatellite analysis (Cordero-Bueso et al., 2011; Le Jeune et al., 2007; Schuller et al., 2007). However, the methodology proposed in Figure 1 enables a large 
number of yeast isolates to be processed, and PFGE is considered a most suitable technique for discriminating between yeast clones (Schuller et al., 2004).

In our laboratory, this technique has been used to characterize the wine yeast population responsible for the spontaneous fermentation of a white wine produced in a winery in SW Spain (Rodríguez et al., 2010). Analyses of industrial-scale fermentations (in 400 000-1 fermentation vessels) were carried out during two consecutive vintages. In 1999 and 2000 a total of 211 and 228 yeast colonies, respectively, from different vessels, were characterised by karyotyping. The degree of polymorphism observed was high, and 17 different karyotypic patterns were detected in 1999, and 21 patterns in 2000. In the two campaigns, we also found patterns belonging to non-Saccharomyces yeasts, the karyotypes of which did not show the four bands running below $500-\mathrm{kb}$. During the fermentation, this population was displaced by S. cerevisiae strains; patterns I, II, III and V were predominant during entire fermentation process in 1999, whereas in 2000 patterns II and V were predominant (see Figure 2). Those were the yeast strains selected for inoculating the industrial fermentations, as will be explained bellow.

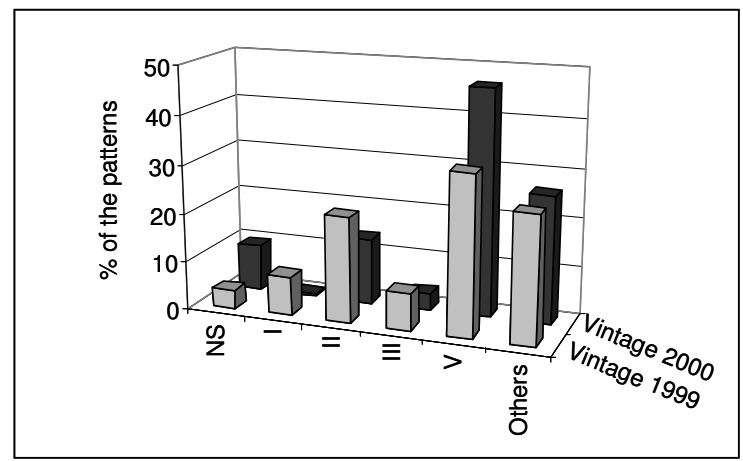

Fig. 2. Frequencies of the majority karyotype patterns (\%) obtained in the spontaneous fermentations of 1999 and 2000. NS corresponds to non-Saccharomyces yeasts.

The results of the characterization of the yeasts also showed that the different strains changed their proportion, and there was a sequential substitution of strains during the fermentation; this gave a valuable indication of the dynamics of the yeasts population throughout the process. Some of these changes were specific to a particular fermentation phase, suggesting that the yeast strains with different electrophoretic karyotypes also differ in their adaptation to the evolving environment at different phases of the fermentation process.

Although the diversity of wild yeast can contribute to high-quality and unique flavour in the finished wine, spontaneous fermentation is often unpredictable and might introduce less desirable traits to the product, sometimes even spoiling a production batch. Other risks associated with spontaneous fermentation include either slow or arrested fermentation. To avoid these problems, winemakers often add cultures of selected yeasts, in the form of active dried yeasts or autochthonous yeasts. Nevertheless, in some cases, these yeasts used as starters are not able to displace the wild yeasts present in the must, since the wild yeasts can be very competitive (Esteve-Zarzoso et al., 2000; Lopes et al., 2007). 
In our work on the analysis of the karyotype, it has been possible to monitor the yeast population under industrial conditions for several years when fermentations of the white wine were inoculated with selected autochthonous yeast strains. This has allowed inoculation strategies to be designed for the correct development of inoculated yeast while retaining the unique regional character of the finished wine (Rodríguez et al., 2010). The strains with patterns I, II, III and V were the most representative during the spontaneous fermentation process (Figure 2) and they could be isolated at the late fermentation phase. These autochthonous yeasts show valuable traits of enological interest, such as high fermentative capacity, ethanol tolerance, and they had a killer phenotype. The capacity of each strain to compete within the mixed population was also tested under semi-industrial conditions by PFGE. The results show that the strains with karyotypes II and V were the most vigorous competitors, followed by the strain with patterns III and I, which were detected in lower proportions (Rodríguez et al., 2010). Therefore, the strains with patterns II, III and V were used to inoculate the fermentations in the year 2001; strains with patterns II and V were used in 2002, 2003 and 2004; and from the year 2005 until the present (2011), only the strain with pattern $\mathrm{V}$ has been used.

The inoculation of industrial vessels of the winery of this study presented several peculiarities. For example: (i) in each vintage year several vessels with a total capacity of 400 000-1 were inoculated; (ii) the inoculums comprising the selected autochthonous yeast strains were prepared from fresh YEPD plates (1\% yeast extract, $2 \%$ glucose, $2 \%$ peptone and $2 \%$ agar) by preparing a starter in which each scaling-up round was performed when the ${ }^{\circ}$ be reached a value between 1-2, and in each round, the fermentation volume was increased tenfold to give high initial levels of inoculum $\left(>60 \times 10^{6}\right.$ viable cells $\left./ \mathrm{ml}\right)$ and ensure the correct development of the inoculated strain; (iii) once the starter cultures were scaled-up and added to a 400 000-1 container, partial volumes were withdrawn and used for the inoculation of other 400 000-1 vessels of the winery; and (iv) the 400 000-1 vessels received random additions of fresh must until reaching the final volume. The frequency and timing of these additions depended on the production yield of fresh must during the vintage campaign.

In our results (Table 1) only the strain with pattern V, called P5, was dominant under industrial conditions and, for this reason, the number of the starter strains were reduced over the years and currently only the strain with karyotype $\mathrm{V}$ is used for the inoculation of the industrial fermentations. In spite of this strain's good capacity for achieving dominance, in some years a high degree of polymorphism was detected in the fermentations; and the cause of the unexpected predominance of wild yeast karyotype was linked to several factors, including: a sudden decrease in the temperature of one of the vessels during the scaling-up process of the inoculation in 2002; the method of inoculation and the scaling-up process, which were changed in 2003, whereby the inoculums of pure culture did not represent $10 \%$ and each scaling-up round was performed when the inoculums had a high sugar content (around 3-5 ${ }^{\circ}$ Bé); and the storage of must in the vessels in which spontaneous fermentation had occurred.

When karyotype $\mathrm{V}$ was dominant in the fermentations, the wine obtained had fruity characteristics, with well-balanced acidity, that satisfied the wine producer. Although we did not obtain a comprehensive aromatic characterization of the wine, the panel of wine tasters (Figure 3) considered the wine produced in these fermentations better than the wine 
obtained either in the spontaneous fermentations of 1999 and 2000 or in the vintage years of 2002 and 2003, when the karyotype V (strain P5) was not detected in high proportion in the yeast population.

\begin{tabular}{llllllll}
\hline Patterns & 2001 & 2002 & 2003 & 2004 & 2005 & 2007 & 2008 \\
\hline II & 7.6 & 9.2 & 3.6 & 0.4 & 0 & 10.7 & 0 \\
III & 8.5 & 0 & 0 & 0 & 0 & 0 & 0 \\
V & 83 & 0.6 & 19.3 & 99.6 & 79.8 & 50 & 100 \\
(NS) & 0.2 & 0 & 0.4 & 0 & 0.4 & 0 & 0 \\
Others & 0.7 & 90.2 & 76.7 & 0 & 19.8 & 39.3 & 0 \\
\hline $\begin{array}{l}\text { Total yeast } \\
\text { analysed }\end{array}$ & 423 & 174 & 223 & 235 & 278 & 142 & 240 \\
\hline
\end{tabular}

Table 1. Frequencies (\%) of the inoculated strains in the industrial fermentations for seven vintage years. This analysis was not performed in the vintage year of 2006. NS: nonSaccharomyces.

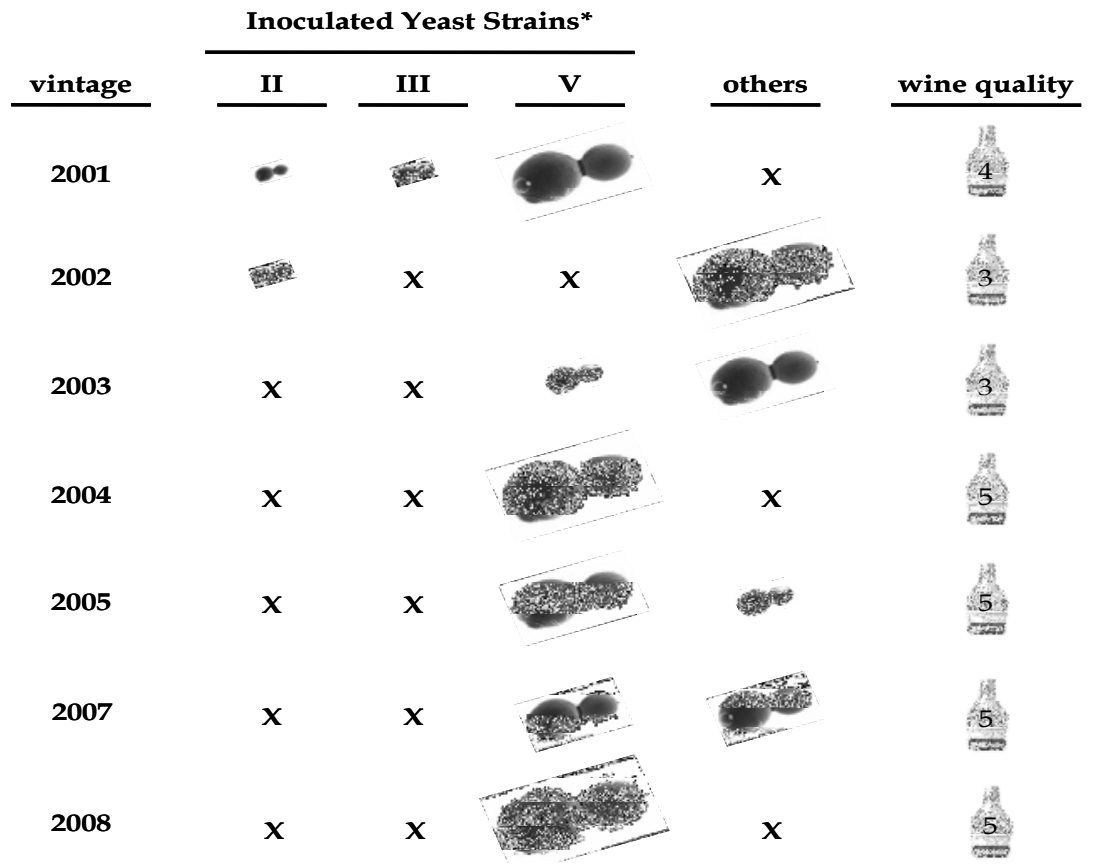

*Strain group II was inoculated in the years 2001 to 2004; the strain with pattern III was inoculated only in 2001; and strain group V was inoculated in all vintages

Fig. 3. Composition of the wine yeast population in each vintage, and its relationship with the quality of the final product. For each year the size of the yeast cell shown is proportional to the contribution of each strain to the total wine yeast population of the winery. The $x$ symbol indicates that the proportion of the strain(s) within the population was below $5 \%$. Wine quality was evaluated by a panel of expert wine-testers from the winery, who graded the final product on a scale from 1 to 5 based on fruity wine with well-balanced acidity desired by the producer ( 5 indicates highest quality and 1 lowest quality). Predominance of the strain with pattern $\mathrm{V}$ corresponded to a better quality of the wine. 
By using PFGE to study the yeast population of the inoculated fermentations, the producer was able to make informed decisions for improving the process; the common factors in the vintages of 2001, 2004, 2005, 2007 and 2008, in which the inoculated strain was dominant, can be highlighted. These factors were the following: (i) the culture was not scaled-up to the next volume until the yeast had fully depleted the sugar to less than 1 ${ }^{\circ}$ Bé (one degree is equivalent to $18 \mathrm{~g} / 1$ of fermentable sugars in the must); therefore all cultures reached a high alcohol content before the addition of fresh must; (ii) the inoculum was always diluted less than 10 -fold in each scaling-up round; (iii) the temperature of the fermentation was kept at $17^{\circ} \mathrm{C}$.

We think that these criteria favoured the adaptation of the inoculums to the conditions of the must obtained in each vintage and to the final conditions within the $400000-1$ industrial vessels. In addition, these criteria favoured the predominance of the inoculated strain with pattern $\mathrm{V}$.

In another study with biodynamic red wines, carried out in the Ribera del Duero D. O. Region (Valladolid, Spain), spontaneous fermentations were also analysed applying PFGE. We studied seven fermentations in three phases during the fermentation process: initial (IF), middle (MF) and final (EF), and 20 isolated strains per sample were characterized by applying PFGE (417 strains in 2008, and 412 strains in 2009). The results for two consecutive vintages studied showed the presence of different types of the yeast during the fermentations that were grouped in three populations. The first population was formed by non-Sacharomyces yeast, whose strains showed patterns with the absence of bands running below the region of $500 \mathrm{~kb}$, which are specific to $S$. cerevisiae strains as reported above. The second population comprised Saccharomyces bayanus var. uvarum (S. uvarum); and the third population included Saccharomyces cerevisiae yeast. The strains of $S$. uvarum were differentiated from the $S$. cerevisiae strains by the presence of two small chromosomes in the region of $245-370 \mathrm{~kb}$, instead of three as for S. Cerevisiae, as reported by Naumov et al. $(2000,2002)$. Non-Sacharomyces (NS) yeasts were dominant in the initial phase of fermentation but were displaced in the subsequent and final phases of the process by another population of yeasts. S. uvarum yeasts were present mainly in the phase mid-way through the fermentation; then the population of $S$. cerevisiae yeasts displaced the NS and S. uvarum yeasts, and remained dominant until the end of the fermentation, in the majority of the deposits analysed. The low frequency of detection of S. uvarum at the end of the fermentation could be indicative of its lower ethanol tolerance compared to $S$. cerevisiae. Within each population yeast strains were also found with different karyotyping patterns, and the distribution (by \%) of these varied in the seven deposits analysed during the two consecutive years studied. Thus, for S. uvarum, considerable variability of strains and a total of 12 different electrophoretic patterns were detected (Figure 4): uI-uVII for vintage 2008; and uI-uIII, $\mathrm{uV}, \mathrm{uVIII}-\mathrm{uXII}$ in 2009. The strains with patterns uI, UII, uIII and uV, followed by UIV (in 2008) and UIX (in 2009) were the most representative in two years studied. Within the population of the $S$. cerevisiae yeasts, the variability of the patterns was higher than in S. uvarum ; 29 (cI-cXXIX) and 27 (cI-cVII, cX-cXII, cXV, cXVII, cXIX, cXXII, cXXIV, cXXX-cXLI) electrophoretic karyotype patterns were detected for 2008 and 2009 respectively. The S. cerevisiae yeast strains most representative of the fermentation process in these years were those that showed the karyotypes cIII, cVI, cXI and cXII. 
The yeast population dynamics presented in this biodynamic red wine were different from those observed in other studies of white wines in which $S$. uvarum was dominant during spontaneous alcoholic fermentation (Demuyter et al., 2004).

Although S. uvarum has been found in other producing regions of the world, such as Alsace (Demuyter et al., 2004), at the moment there are no studies about the population dynamics of S. uvarum in Ribera del Duero, Spain.

The use of the PFGE technique allows analysts to detect a high degree of polymorphism in the population of the yeast and to monitor the dynamics of yeast ecology during the fermentation; this is because it is able to show the occurrence of gross chromosomal rearrangements, which is the phenomenon that mainly accounts for the rapid evolution of yeast clones subjected to industrial conditions (Infante et al., 2003). The technique also shows the most representative yeast strains in the industrial winemaking process, which are partially but significantly responsible for the finished wine's quality. Knowledge of these main strains can be used as a criterion for making a first selection of the autochthonous yeast. Later, with these previously selected strains, PFGE can be applied to study the features of these strains that are of enological interest, as described in recent years, which fall into three main categories: (i) properties that affect the performance of the fermentation process; (ii) properties that determine the quality of the wine; and (iii) properties associated with the commercial production of yeast (reviewed in Fleet, 2008). The yeasts selected by these means can then be used for inoculation in the fermentation process, thus improving winemaking.

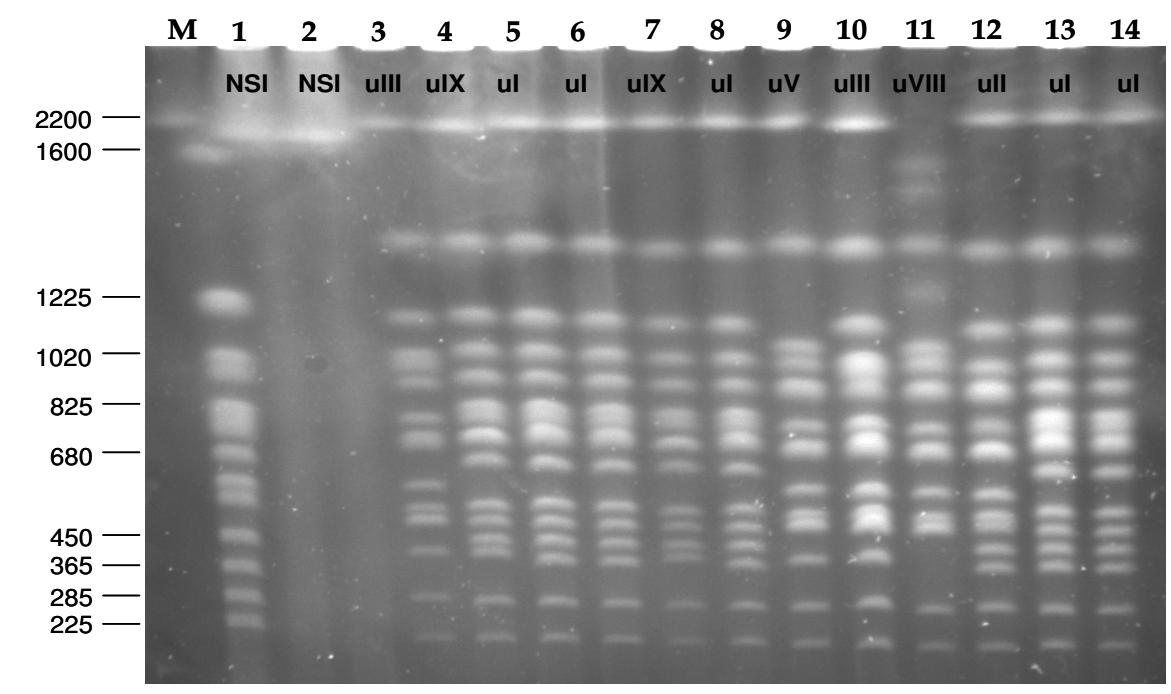

Fig. 4. Electrophoretic karyotype of 14 colonies isolated from the sample taken in 2009 from a vessel in the phase mid-way through the fermentation process. Colonies 3-14 correspond to different karyotype patterns (uI-uIX) of yeast strains found in the S. uvarum species. Isolates 1-2 correspond to non-Saccharomyces strains which show the same pattern (NSI), with absence of bands running below $1225 \mathrm{~kb}$. The chromosomes of the S. cerevisiae YNN295 strain were used as reference $(\mathrm{M})$. 


\section{Application of mtDNA-RFLP as a rapid method for monitoring the inoculated yeast strains in wine fermentations}

Although PFGE has been reported to be the most efficient in discriminating between different strains of $S$. cerevisiae, the mtDNA-RFLP technique is frequently used to differentiate between yeast isolates of the same species (González et al., 2007) because it enables a larger number of strains to be analyzed in a shorter time; it is a fast, simple, reliable and economic method, which does not require sophisticated material or specialized personnel (Fernández-Espinar et al., 2006). For these reasons, it is a very suitable technique for use by industry.

Most of the mitochondrial DNA in yeasts does not code proteins, and contains a high proportion of AT bases. Analysts can take advantage of this characteristic to characterize yeasts; it involves measuring variation in sequences in the mtDNA affecting the restriction sites of several endonucleases. Endonucleases such as AluI, Hinfl or RsaI, recognise the very frequent restrictions in the chromosomal DNA but not in the mitochondrial DNA, leading to a total cleavage of the chromosomal DNA in small pieces. These pieces can be easily differentiated from the mitochondrial fragments, which appear as bands with an electrophoretic mobility corresponding to molecules greater than $2 \mathrm{~kb}$, generating polymorphisms that allow the characterization between yeast strains.

In previous studies applying this technique it has been demonstrated that the population of a fermentation vessel is "taken over" by wild yeasts, which displace the inoculated yeast strain, reducing it to a minority presence (Esteve-Zarzoso et al., 2000; Lopes et al., 2007; Raspor et al., 2002). In our research, when we have analyzed the inoculated fermentation of white wine as described above, we have found several examples of real situations that led to a significant decrease in the proportion of the inoculated strain (pattern V) and, in consequence, the quality of the wine was reduced. In order to minimize the impact of unwanted yeasts, wineries need a simple method for rapid diagnosis of the degree of dominance of inoculated strains, a method that could be performed routinely during the fermentation process (Ambrona et al., 2006, López et al., 2003). With this object we have used RFLP analysis of mtDNA for the rapid monitoring of the dominance, or otherwise, of inoculated yeast strains in industrial fermentations of white and red wines in a winery in southern Spain (Rodriguez et al., 2011).

We apply this technique directly to samples of fermenting wine without previously isolating yeast colonies. For white wine fermentations, a rapid assay is performed consisting of taking a sample of fermenting must, purifying the DNA from harvested cells, and obtaining the restriction patterns by digestion with endonuclease Hinfl. The same protocol is applied to red wine fermentation, but an overnight cultivation step is added before purification of the DNA (Figure 5).

The criterion for considering the result of the rapid test to be positive was obtaining restriction patterns of mtDNA that were identical for the total cells and the inoculated strain; when this is the case, the starter yeast can be taken as being dominant in the fermentation. The result was considered negative when additional bands, or absence of bands, were observed in the patterns; in this case neither the dominance, nor even the presence, of the inoculated yeast strain can be assured. 


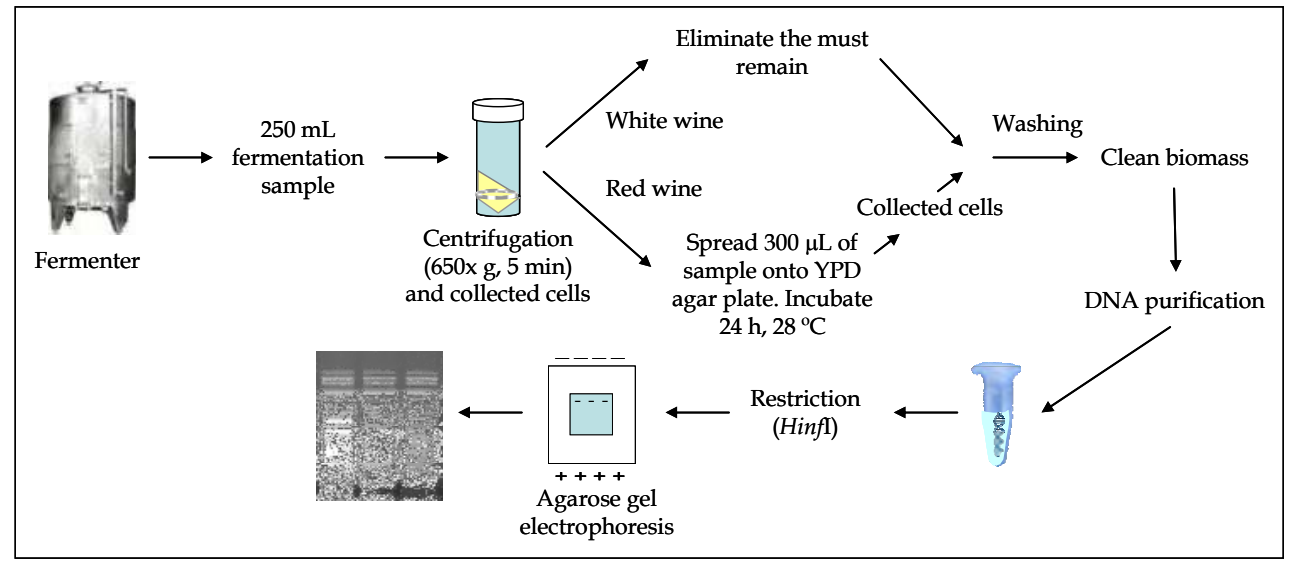

Fig. 5. Methodology for the rapid test assays by restriction analysis of the mtDNA.

This methodology of the RFLP test has been applied since 2005 in a winery of SW Spain and the results were obtained 11 and 23 hours after sampling, for white and red wine respectively (Rodríguez et al., 2011). If the wine-producer knows whether or not the presence of the inoculated yeasts has suffered a sudden decrease, and the inoculated strain is no longer dominant, in any phase of the fermentation process, a rapid intervention can be made. Since this year the winery has only used the selected autochthonous strain P5 for the inoculation of its white wine fermentations; this strain was in a clear majority in spontaneous fermentations, as reported above, and it shows karyotype pattern V. The peculiarities of the inoculation of industrial vessels for this wine have been described above (section 2). After applying the RFLP test, the correct course of the fermentation of all starters was assured before the inoculation of the industrial vessels. Generally, all the fermentations are tested in at least two different phases of the process: first after refills with fresh must and again when the fermentations are finished.

In addition, the results were checked using PFGE to validate the previous results obtained from the RFLP test. For this validation, 34 samples tested by RFLP were analyzed by electrophoretic karyotype of 323 colonies for white wine. The results indicated that when RFLP test was positive, the inoculated strain was present in the fermentation at $64 \%$ or more. When the RFLP tests were negative it was confirmed by PFGE that the starter yeast was present at only $60 \%$ or less.

The red wine of the winery was fermented in stainless steel vessels of 27.0001 and no refills of must were carried out. The fermentations were inoculated with several commercial active dried wine yeasts (ADWY) by hydration following manufacturer's instructions. For this wine, for 331 colonies analyzed by PFGE, the results of the RFLP test correctly predicted the results obtained later by applying PFGE. However, in this case, the limit found for white wine cannot be established because, from the positive results obtained by applying the RFLP test, the presence of the inoculated strain was greater than $75 \%$, and all the negative results were at $55 \%$ or less (Rodríguez et al., 2011). Nevertheless, further experiments will be necessary to confirm these correlations because the RFLP test shows qualitative results and the actual percentage implantation of the starter yeast cannot be known when the results are 
positive or negative. Figure 6 shows examples of positive and negative results for the last two years (2009 and 2010) for the dominance and non-dominance respectively of the inoculated strain P5 in white wine of the same winery described above.

For the 2009 vintage, we tested several different vessels at the initial phase of the fermentation, for white wine (Panel A). The results were positive for all cases after applying the rapid test, i.e. the restriction patterns of the samples and the inoculated strain were identical, and the strain P5 was responsible at the beginning of the fermentation process displacing other wild yeasts.

A

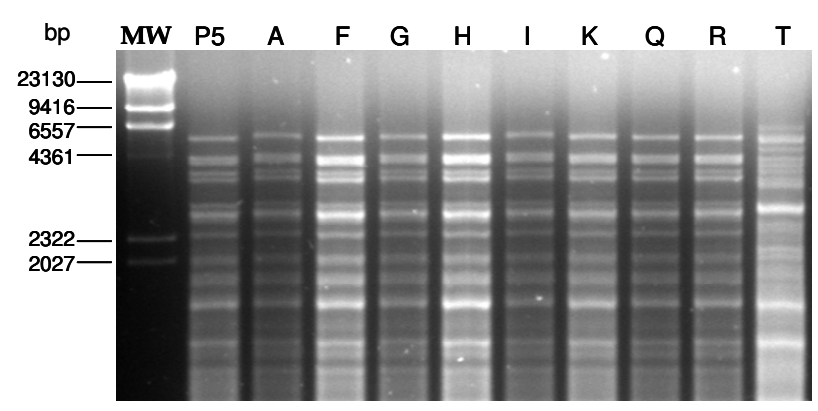

B

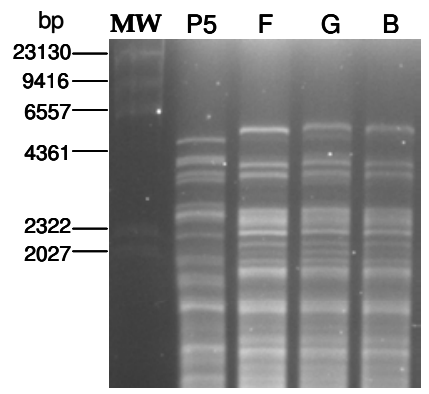

Fig. 6. Rapid test based on mtDNA-RFLP with Hinfl of samples from white wine fermentations which were inoculated with autochthonous yeast strain P5. Panel A shows results of samples taken in 2009 from vessels $A, F, G, H, I, K, Q, R$ and $T$ at the initial fermentation phase (11-7 $\left.{ }^{\circ} \mathrm{Bé}\right)$. Panel $\mathrm{B}$ shows results of samples taken in the 2010 vintage from vessels F, G and B during the main phase of the fermentation ( $\left.5-3^{\circ} \mathrm{Bé}\right)$. MW is the lambda-HindIII molecular marker.

Only the fermentation vessel reference $\mathrm{T}$ was considered negative for the RFLP test at the beginning of the process. In this vessel evidence was observed of spontaneous fermentation before the inoculation, due to the conditions in which the must was stored. When this must was inoculated, the strain P5 did not implant successfully and it was concluded that another wild yeast population was dominant.

In situations like this, the winemaker can take the decision not to use the fermenting must for the inoculation of other vessels.

Panel B (Figure 6) shows examples of the negative results of the RFLP test in three vessels mid-way during the fermentation process. In these deposits the population was perhaps similar because the restriction pattern of the total cells in each sample was similar. After the vintage, 20 colonies were isolated from the same sample previously analyzed by rapid test in the vessel G (Figure 6, panel B) in order to confirm the composition of the yeast population by karyotype. Surprisingly, all the clones show the same pattern as a commercial yeast strain used for the inoculation in the fermentation of another type of white wine in the previous year. It is assumed that this commercial yeast was also dominant in the fermentations sampled in vessels $\mathrm{F}$ and $\mathrm{B}$. In previous studies researchers have reported the risks in using commercial yeast, because they can become part of the microbiota of the 
winery, effectively creating their own ecosystem, and can subsequently be predominant in the fermentations (Santamaría et al., 2005). In our study, we think that this commercial yeast was present in equipment which was not properly cleaned. When the wine-producer was more careful in the next vintage, there were no problems of contaminations by commercial yeast, and the dominant yeast in the fermentations was the inoculated autochthonous yeast P5 (data not shown). Therefore, it was confirmed that the commercial yeast had not acquired an ecological niche because it presumably did not adapt well to the ecosystem of a properly-cleaned winery.

As stated, the results of the RFLP can be obtained 11 and 23 hours after taking the sample for white and red wine respectively. However, this time can be shortened further, because it depends on the method used to rupture cells, on the number of samples analyzed per day, and on whether the samples contain a greater amount of must residues. In the case of red wine, there was another problem in shortening the test time, because the residues were difficult to clear by centrifugation; we think that some compounds remaining in the digested DNA samples were inhibitory for the endonuclease. Therefore, a step has been added in the protocol of the rapid test (Figure 5) in which the sample of the red must is plated on YPDagar and incubated overnight at $28^{\circ} \mathrm{C}$. Nevertheless, we think that, for red wine, the time taken to obtain the results could also be shortened further, like that for white wine, if the clean biomass can be separated from the must residues in a few minutes. To achieve this, further experiments will need to be carried out.

\section{Relevance of proteomic analysis in the winemaking process}

In brief, proteomics can be described as a set of techniques for unravelling complex mixtures of proteins. In spite of it being a relatively recent technique, most of the systems used are widely known by the research community. However, the crucial work for its final "take-off" as a viable technique has been the modifications made to the mass spectrometry system, to allow the analysis of peptides and proteins. The exponential growth in the number of entries for genes and/or proteins in the databases now makes protein analysis and identification much easier, as well. This, combined with the use of powerful methods of fractionation and separation of peptides and proteins, such as 2D-PAGE (two dimensional polyacrylamide electrophoresis) and high resolution liquid chromatography, proteomics has been consolidated since the mid- 90 's, as the science for massive protein analysis; it is now the main methodology for unravelling biological processes, leading some authors to describe the current period as the "post-genomic era".

Proteomics has been defined as the set of techniques for studying the complex mixture of proteins, named the Proteome, that exists in any specific cell, microorganism, tissue, etc, used in specific experimental conditions, culture, sampling, etc. It is a highly dynamic system, and is more complex than genomics because, while the genome of an organism is more or less constant, the number of proteomes obtained from a specific genome is infinite. It depends on the assayed cell, tissue, culture conditions, etc., because each change produces a modification in the observed proteome. An additional factor of complexity derives from the fact that there are changes that occur in proteome that are not encoded in the genome. These changes mainly originate from two sources: (i) the editing of the mRNA; and (ii) posttranslational modifications (PTMs) that normally serve to modify or modulate the activity, function or location of a protein in different physiological or metabolic contexts. More than 
200 different PTMs have been described (including phosphorylation, methylation, acetylation, etc.) that transform each single gene into tens or hundreds of different biological functions. Before the advances made in proteomics, the differential analysis of the genes that were expressed in different cell types and tissues in different physiological contexts was done mainly through analysis of mRNA. However, for wine yeast, it has been proved that there is no direct correlation between mRNA transcripts and protein content (Rossignol et al., 2009). It is known that mRNA is not always translated into protein, and the amount of protein produced by a given amount of mRNA depends on the physiological state of the cell. Proteomics confirms the presence of the protein and provides a direct measure of its abundance and diversity.

In terms of methodology, proteomics approaches are classified in two groups: (i) gel-free systems based on the use of various chromatography methods; and (ii) gel-based methods that use mainly two-dimensional polyacrylamide gel electrophoresis (2DE). This latter approach will form the focus of our discussion here, given the subject matter of this book. As a succinct summary, the typical workflow of a proteomic experiment begins with the experimental design. This must be studied in depth, and it will delimit the conclusion obtained, even more so when comparisons are made between two strains, cultures or physiological stages, among others. As an optimum, only one factor among the various different assayed conditions must change (Fernandez-Acero et al., 2007). Several biological replicates, usually from 3 to 5, will be required depending on the strategy adopted. The next key step is to obtain a protein extract of high enough quality to separate complex mixtures of proteins. Usually, the protein extraction is done in sequential steps. First the tissue, cells, etc. are ruptured using mechanical or chemical techniques. Then, proteins are precipitated and cleaned. Most existing protocols use acetone and trichloroacetic acid. In the next step the proteome is defined and visualized using electrophoretic techniques. 2DE has been widely used for this purpose. Using this technique, proteins are separated using two different parameters. In the first dimension, proteins from the purified extract are separated by their iso-electric point using an iso-electrofocusing (IEF) device. Then, the focused strips are loaded in a polyacrylamide gel where the proteins are separated by their molecular weight. This system allows the separation of hundreds of proteins from one complex mixture. The gels are visualized with unspecific (Comassie, Sypro, etc.) or specific (e.g. Phospho ProQ diamond) protein stains. The gels are digitalized and analyzed with specific software to reveal the significant spots. These spots are identified using mass spectrometry; commonly, for 2DE approaches, MALDI TOF/TOF is used. The huge list of identified proteins obtained is studied to discover the biological relevance of each identification.

In spite of the many achievements of proteomics, only a few proteomic studies have been carried out on wine yeast, whereas mRNA expression has been widely used to study a broad range of industrial conditions. However, Rossignol et al. (2009) show that substantial changes in protein levels during alcoholic fermentation are not directly associated with changes in the transcriptome; this suggests that the mRNA is selectively processed, degraded and/or translated. This conclusion is important: it is the proteome, not the genome nor the transcriptome, that is the relevant level of analysis for understanding the adaptations of wine yeasts during alcoholic fermentation, since these are responsible for the phenotype.

The usual strategy for wine production is the inoculation of selected yeast strains into the must, decreasing the lag phase, a quick and complete fermentation of the must, and a high 
degree of reproducibility of the final product. The development of global analysis methodology has allowed a detailed analysis to be made of changes in gene expression and protein levels at various time-points during vinification. Zuzuarregui et al. (2006) presented a comparison between the mRNA and protein profiles of two yeast strains with different fermentation behaviours, which correlates with divergence in the fermentation profiles. The results indicate changes in the mRNA and protein levels and, probably, post-translational modifications of several proteins, some of them involved in stress response and metabolism.

Another proteomic approach was aimed at studying the adaptation of a wild-type wine yeast strain, isolated from a natural grape must, to physiological stresses during spontaneous fermentation (Trabalzini et al., 2003). Using 2DE, changes in the yeast proteome were monitored during glucose exhaustion, before the cells begin their stationary phase. The proteome adaptation of $S$. cerevisiae seems to be directed or caused by the effects of ethanol, leading to both hyperosmolarity and oxidative responses. Through the use of a wild-type S. cerevisiae strain and PMSF, which is a specific inhibitor of vacuolar proteinase B, it was also possible to distinguish the specific contributions of the vacuole and the proteasome autoproteolytic process. This is the first study that follows the adaptation of a physiologically wild wine yeast strain progressively to the exhaustion of an essential nutrient, glucose.

To monitor yeast stress Salvadó et al. (2008), using ADWY (active dried wine yeast) inoculated into the must, have observed its behaviour in different stress situations, i.e. high sugar concentration or low $\mathrm{pH}$. The main responses after inoculation in a fermentable medium were the activation of several genes of the fermentation pathway and the monoxidative branch of the pentose pathway, and the induction of a huge cluster of genes related to ribosomal biogenesis and protein synthesis. The changes that occur during the lag phase are characterized by an overall change in the protein synthesis and reflect the physiological conditions of the yeast, which affects the fermentative capacity and fermentation performance. Certain enological practices increase these stressful conditions for ADWY. This is the case of low-temperature fermentation, which improves taste by restructuring flavour profiles, with potential enological applications. This study focuses on changes that occur in ADWY after inoculation in a synthetic wine. These changes reflect adaptation to a new medium.

Previous reports have shown that proteomic analysis of wine yeast is the most relevant tool for understanding the physiological changes involved in winery processes. The information obtained may improve the quality of the final product. Our group has been a pioneer in fungal proteomic approaches (Fernandez-Acero et al., 2007, 2011; Garrido et al., 2010), and in line with this, we are now exploring the relevance of proteomics in wine improvement (Muñoz-Bernal et al., 2011). Our group has developed new protocols for obtaining the proteome and subproteomes of yeast, and the results to date suggest that there is a lot of biological information to be studied and analyzed from the proteomic perspective. The relevance of this achievement for winery processes could be significant.

\section{Conclusions}

Application of the PFGE technique allows the yeast population in the wine fermentation process to be characterized. The technique has been reported to be the most efficient for 
discriminating between S. cerevisiae yeast clones (Schuller et al., 2004), and it differentiates these from the specie $S$. bayanus var. uvarum (Naumov et al., 2002). It is also able to reveal the occurrence of gross chromosomal rearrangements, which account for the rapid evolution shown by yeast in industrial environments (Infante et al., 2003). Using PFGE, we have detected a high degree of polymorphism in the population of spontaneous fermentations of different types of wine produced in different regions of Spain, and it was observed that there were yeast strains that were specific to a particular phase of the fermentation process. This suggests that yeast strains with different karyotypes also differ in their adaptation to the evolving environment at different phases of the fermentation process. Studies for the molecular characterization of wine yeast represent a first step for selecting autochthonous yeast strains which are better adapted to specific conditions of a particular wine-making region. Moreover, such knowledge in respect of yeast populations may lead to the identification of a new natural source of wine yeast that could be used by the industry in the future as a new commercial starter (Fleet, 2008).

Studies by PFGE of the yeast population in inoculated fermentations also allow producers to understand and make informed decisions for improving their processes. Our results suggest that the success of the inoculation protocol is highly dependent on adequate preparation of the inoculums, which must facilitate the adaptation of the inoculated strains to the final conditions of the fermentation.

The RFLP test designed to monitor and confirm that the population of the inoculated yeast has reached and maintained predominance, in white or red wines, is proposed as a response to one of the major challenges for microbiological control in the wine industry. In our results real situations are shown taking place during actual wine fermentations; for example spontaneous fermentations sometimes occur before the inoculation. We offer a test which the winemaker can use to obtain a reliable indication of whether or not wild yeasts are displacing the inoculated strains. If the strategy presented is followed, the wine producer would be able to identify and correct in time the unwanted evolution of the yeast population - usually by re-inoculating the selected strains and/or correcting a deviation in temperature or change in some other parameter of the vessel that might have caused the unwanted situation.

Our studies are among the first examples carried out at the industrial scale showing how molecular techniques can be successfully applied to improve quality and efficiency in the winemaking process.

Despite the achievements already made, we are also exploring the potential use of the latest molecular proteomics techniques to unravel the biological component of the complex winemaking processes. Proteomics data collected to date strongly suggest that these techniques are potentially very useful for controlling the fermentation process and for assuring the quality of the finished wine; they offer excellent prospects for improving these processes in the near future.

\section{Acknowledgements}

This work was supported by grants PETRI 95-0855 OP from the DGICYT of the Ministry of Science and Innovation, and OT 054/174/015/020/114/136/104 from Bodegas Barbadillo S.L. of Sanlúcar de Barrameda, Spain, and CDTI-IDI-20101408. 


\section{References}

Ambrona, J., Vinagre, A., Maqueda, M., Álvarez, M. L., Ramirez, M. (2006). Rhodamine-pink as a genetic marker for yeast populations in wine fermentation. Journal of Agricultural and Food Chemistry, Vol. 54, pp. 2977-2984, ISSN 0021-8561

Carle, G. F., Olson, M. V. (1985). An electrophoretic karyotype for yeast. Proceedings of the National Academy of Sciences of the United States of America, Vol. 82, pp. 3756-3760, ISSN 0027-8424

Chu, G., Vollarath, D., Davis, R. W. (1986). Separation of large DNA molecules by contourclamped homogeneous electric field. Science, Vol. 234, pp. 1582-1585, ISSN 00368075

Codón, J. M., Benítez, T., Korhola, M. (1998). Chromosomal polymorphism and adaptation to specific industrial environments of Sacharomyces strains. Applied Microbiology and Biotechnology, Vol. 49, pp. 154-163, ISSN 0175-7598

Cordero-Bueso, G., Arroyo, T., Serrano, A., Tello, J., Aporta, I., Vélez, M. D., Valero, E. (2011). Influence of the farming system and vine variety on yeast communities associated with grape berries. International Journal of Food Microbiology, Vol. 145, pp. 132-139, ISSN 0168-1605

Demuyter, C., Lollier, M., Legras, J. L., Le Jeune, C. (2004). Predominance of Saccharomyces uvarum during spontaneous alcoholic fermentation, for three consecutive years, in an Alsatian winery. Journal of Applied Microbiology, Vol. 97, pp. 1140-1148, ISSN 1364-5072

Esteve-Zarzoso, B., Gostíncar, A., Bobet, R., Uruburu, F., Querol, A. (2000). Selection and molecular characterization of wine yeast isolated from the 'El Penedès' area (Spain). Food Microbiology, Vol. 17, pp. 553-562, ISSN 0740-0020

Fernández-Acero, F. J., Carbú, M., Garrido, C., Vallejo, I., Cantoral, J. M. (2007). Proteomic Advances in Phytopathogenic Fungi. Current Proteomics, Vol. 4, No. 2, pp. 79-88, ISSN 1570-1646

Fernández-Acero, F. J., Carbú, M., El-Akhal, M. R., Garrido, C., González-Rodríguez, V. E., Cantoral, J. M. (2011). Development of Proteomics-Based Fungicides: New Strategies for Environmentally-Friendly Control of Fungal Plant Diseases. International Journal of Molecular Sciences, Vol. 12, pp. 795-816, ISSN 1422-0067

Fernández-Espinar, M. T., Martorell, P., De Llanos, R., Querol, A. (2006). Molecular methods to identify and characterize yeasts in foods and beverages. In: Querol, A., Fleet, G. H. (Eds), The Yeast Handbook: Yeast in Food and Beverages, Springer, pp. 55-82

Fleet, G.H. (2008). Wine yeasts for the future. FEMS Yeast Research, Vol. 8, pp. 979-995, ISSN 1567-1356

Garrido, C., Cantoral, J. M., Carbú, M., González-Rodríguez, V. E., Fernández-Acero, F. J. (2010). New Proteomic Approaches to Plant Pathogenic Fungi. Current Proteomics, Vol. 7, pp. 306-315, ISSN 1570-1646

González, S. S., Barrio, E., Querol, A. (2007). Molecular identification and characterization of wine yeast isolated from Tenerife (Canary Island, Spain). Journal of Applied Microbiology, Vol. 102, pp. 10185-1025, ISSN 1364-5072

Infante, J.J., Dombek, K.M., Rebordinos, L., Cantoral, J.M. \& Young, E.T. (2003). Genomewide caused chromosomal rearrangements play a major role in the adaptive evolution of natural yeast. Genetics, Vol. 165, pp. 1745-1759, ISSN 0016-6731 
Le Jeune, C., Lollier, M., Demuyter, C., Erny, C., Legras, J. L., Aigle, M., Masneuf-Pomarède, I. (2007). Characterization of natural hybrids of Saccharomyces cerevisiae and Saccharomyces bayanus var. uvarum. FEMS Yeast Research, Vol. 7, pp. 540-549, ISSN 1567-1356

Lopes, C. A., Rodríguez, M. E., Sangorrín, M., Querol, A., Caballero, A. C. (2007). Patagonian wines: implantation of an indigenous strain of Saccharomyces cerevisiae in fermentations conducted in traditional and modern cellars. Journal of Industrial Microbiology \& Biotechnology, Vol. 34, pp. 139-149, ISSN 1367-5435

Lopez, V., Frenández-Espinar, M. T., Barrio, E., Ramón, D., Querol, A. (2003). A new PCRbased method for monitoring inoculated wine fermentations. International Journal of Food Microbiology, Vol. 81, pp. 63-71, ISSN 0168-1605

Martínez, M., Gac, S., Lavin, A., Ganga, M. (2004). Genomic characterization of Saccharomyces cerevisiae Straits isolated from wine-producing areas in South America. Journal of Applied Microbiology, Vol. 96, pp. 1161-1168, ISSN 1364-5072

Mesa, J. J., Infante, J. J., Rebordinos, L., Cantoral, J. M. (1999). Characterization of yeast involved in the biological ageing of Sherry wines. LWT Food Science and Technology, Vol. 32, pp. 114-120, ISSN 0023-6438

Mesa, J. J., Infante, J. J., Rebordinos, L., Sánchez, J. A., Cantoral, J. M. (2000). Influence of the yeast genotypes on enological characteristics of Sherry wines. American Journal of Enology and Viticulture, Vol. 51, 1, pp. 15-21, ISSN 0002-9254

Mortimer, R.K. (2000). Evolution and variation of the yeast (Saccharomyces) genome. Genome Research, Vol. 10, pp. 403-409, ISSN 1088-9051

Muñoz-Bernal, E., Fernández-Acero, F. J., Rodríguez, M. E., Cantoral, J. M. (2011). Variación en el perfil 2DE en Saccaromyces bayanus var. uvarum inducido por temperatura. Análisis diferencial de proteoma. XXIII Congreso Nacional de Microbiología, Salamanca (Spain), 11-14 July 2011

Naumov, G. I., Masneuf, I., Naumova, E. S., Aigle, M., Dubourdieu, D. (2000). Association of Saccharomyces bayanus var. uvarum with some French wines: genetics analysis of yeast populations. Research in Microbiology, Vol. 151, pp. 683-691, ISSN 0923-2508

Naumov, G. I., Naumova, E. S., Antunovics, Z., Sipiczki, M. (2002). Saccharomyces bayanus var. uvarum in Tokaj wine-making of Slovakia and Hungary. Applied Microbiology and Biotechnology, Vol. 59, pp. 727-730, ISSN 0175-7598

Raspor, P., Cus, F., Povhe Jemec, K., Zagorc, T., Cadez, N., Nemanic, J. (2002). Yeast population dynamics in spontaneous and inoculated alcoholic fermentations of Zametovka must. Food Technology and Biotechnology, Vol. 40, pp. 95-102, ISSN 13309862

Rodríguez, M.E., Infante, J.J., Molina, M., Domínguez, M., Rebordinos, L. \& Cantoral, J.M. (2010). Genomic characterization and selection of wine yeast to conduct industrial fermentations of a white wine produced in a SW Spain winery. Journal of Applied Microbiology, Vol. 108, pp. 1292-1302, ISSN 1364-5072

Rodríguez, M.E., Infante, J.J., Molina, M., Rebordinos, L. \& Cantoral J.M. (2011). Using RFLP-mtDNA for the rapid monitoring of the dominant inoculated yeast strain in industrial wine fermentations. International Journal of Food Microbiology, Vol. 145, pp. 331-335, ISSN 0168-1605 
Rossignol, T., Kobi, D., Jacquet-Gutfreund, L. \& Blondin, B. (2009). The proteome of a wine yeast strain during fermentation: correlation with the transcriptome. Journal of Applied Microbiology, Vol. 107, pp. 47-55, ISSN 1364-5072

Salvadó, Z., Chiva, R., Rodríguez-Vargas, S., Rández-Gil, F., Mas, A., Guillamón, J. M. (2008). Proteomic evolution of a wine yeast during the first hours of fermentation. FEMS Yeast Research, Vol. 8, pp. 1137-1146, ISSN 1567-1356

Santamaría, P., Garijo, P., López, R., Tenorio, C., Gutiérrez, A. R. (2005). Analysis of yeast population during spontaneous alcoholic fermentation: Effect of the age of the cellar and the practice of inoculation. International Journal of Food Microbiology, Vol. 103, pp. 49-56, ISSN 0168-1605

Schuller, D., Valero, E., Dequin, S. \& Casal, M. (2004). Survey of molecular methods for the typing of wine yeast strains. FEMS Microbiology Letters, Vol. 231, pp. 19-26, ISSN 1567-1356.

Schuller, D., Casal, M. (2007). The genetic structure of fermentative vineyard-associated Saccharomyces cerevisiae populations revealed by microsatellite analysis. Antonie van Leeuwenhoek, Vol. 91, pp. 137-150, ISSN 0003-6072

Trabalzini, L., Paffetti, A., Scaloni, A., Talamo, F., Ferro, E., Coratz, G., Bovalini, P., Santucci, A. (2003). Proteomic response to physiological fermentation stresses in a wild-type wine strain of Sacharomyces cerevisiae. Biochemistry Journal, Vol. 370, pp. 35-46

Viana, F., Gil, J. V., Vallés, S., Manzanares, P. (2009). Increasing the levels of 2-phenyl acetate in wine through the use of a mixed culture of Hanseniaspora osmophila and Saccharomyces cerevisiae. International Journal of Food Microbiology, Vol. 135, pp. 68-74, ISSN 0168-1605

Zuzuarregui, A., Monteoliva, L., Gil, C., del Olmo, M. (2006). Transcriptomic and proteomic approach for understanding the molecular basis of adaptation of Saccharomyces cerevisiae to wine fermentation. Applied and Environmental Microbiology, Vol. 72, No. 1, pp. 836-847, ISSN 0099-2240 


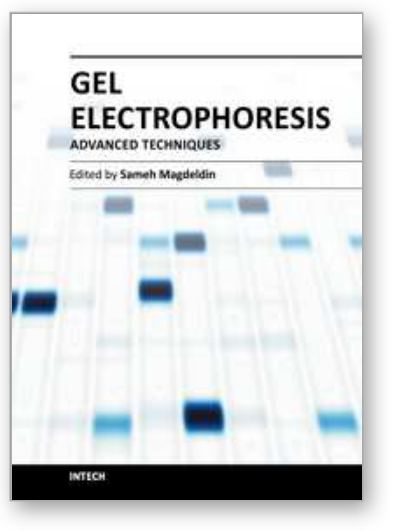

\author{
Gel Electrophoresis - Advanced Techniques \\ Edited by Dr. Sameh Magdeldin
}

ISBN 978-953-51-0457-5

Hard cover, 500 pages

Publisher InTech

Published online 04, April, 2012

Published in print edition April, 2012

As a basic concept, gel electrophoresis is a biotechnology technique in which macromolecules such as DNA, RNA or protein are fractionated according to their physical properties such as molecular weight or charge. These molecules are forced through a porous gel matrix under electric field enabling uncounted applications and uses. Delivered between your hands, a second book of this Gel electrophoresis series (Gel Electrophoresis- Advanced Techniques) covers a part, but not all, applications of this versatile technique in both medical and life science fields. We try to keep the contents of the book crisp and comprehensive, and hope that it will receive overwhelming interest and deliver benefits and valuable information to the readers.

\title{
How to reference
}

In order to correctly reference this scholarly work, feel free to copy and paste the following:

María Esther Rodríguez, Laureana Rebordinos, Eugenia Muñoz-Bernal, Francisco Javier Fernández-Acero and Jesús Manuel Cantoral (2012). Application of Gel Electrophoresis Techniques to the Study of Wine Yeast and to Improve Winemaking, Gel Electrophoresis - Advanced Techniques, Dr. Sameh Magdeldin (Ed.), ISBN: 978-953-51-0457-5, InTech, Available from: http://www.intechopen.com/books/gel-electrophoresis-advancedtechniques/application-of-gel-electrophoresis-techniques-to-the-study-of-wine-yeast-and-to-improvewinemaking

\section{INTECH}

open science | open minds

\author{
InTech Europe \\ University Campus STeP Ri \\ Slavka Krautzeka 83/A \\ 51000 Rijeka, Croatia \\ Phone: +385 (51) 770447 \\ Fax: +385 (51) 686166 \\ www.intechopen.com
}

\author{
InTech China \\ Unit 405, Office Block, Hotel Equatorial Shanghai \\ No.65, Yan An Road (West), Shanghai, 200040, China \\ 中国上海市延安西路65号上海国际贵都大饭店办公楼405单元 \\ Phone: +86-21-62489820 \\ Fax: +86-21-62489821
}


(C) 2012 The Author(s). Licensee IntechOpen. This is an open access article distributed under the terms of the Creative Commons Attribution 3.0 License, which permits unrestricted use, distribution, and reproduction in any medium, provided the original work is properly cited. 